\title{
Dissociating the Role of Prefrontal and Premotor Cortices in Controlling Inhibitory Mechanisms during Motor Preparation
}

\author{
Julie Duque, ${ }^{1,2,3}$ Ludovica Labruna, ${ }^{1,2}$ Sophie Verset, ${ }^{3}$ Etienne Olivier, ${ }^{3}$ and Richard B. Ivry ${ }^{1,2}$ \\ ${ }^{1}$ Department of Psychology and ${ }^{2}$ Helen Wills Neuroscience Institute, University of California, Berkeley, California 94720, and ${ }^{3}$ Institute of Neuroscience, \\ Laboratory of Neurophysiology, Université catholique de Louvain, B-1200 Brussels, Belgium
}

Top-down control processes are critical to select goal-directed actions in flexible environments. In humans, these processes include two inhibitory mechanisms that operate during response selection: one is involved in solving a competition between different response options, the other ensures that a selected response is initiated in a timely manner. Here, we evaluated the role of dorsal premotor cortex (PMd) and lateral prefrontal cortex (LPF) of healthy subjects in these two forms of inhibition by using an innovative transcranial magnetic stimulation (TMS) protocol combining repetitive TMS (rTMS) over PMd or LPF and a single pulse TMS (sTMS) over primary motor cortex (M1). sTMS over M1 allowed us to assess inhibitory changes in corticospinal excitability, while rTMS was used to produce transient disruption of PMd or LPF. We found that rTMS over LPF reduces inhibition associated with competition resolution, whereas rTMS over PMd decreases inhibition associated with response impulse control. These results emphasize the dissociable contributions of these two frontal regions to inhibitory control during motor preparation. The association of LPF with competition resolution is consistent with the role of this area in relatively abstract aspects of control related to goal maintenance, ensuring that the appropriate response is selected in a variable context. In contrast, the association of PMd with impulse control is consistent with the role of this area in more specific processes related to motor preparation and initiation.

\section{Introduction}

Human competence entails the fluid navigation through continuous sets of action choices. We assume that, in the mature state, the neural system has evolved to allow us to select actions that have the highest likelihood of achieving our goals in a given context. Top-down control mechanisms are essential for guiding such goal-oriented behaviors (Rushworth et al., 2009; Cisek and Kalaska, 2010; Cai et al., 2011). These mechanisms involve a fine interplay between excitatory and inhibitory processes.

Here, we build on evidence implicating the operation of two inhibitory mechanisms that act concurrently, yet with different computational purposes, during response preparation (Duque et al., 2010). One mechanism is associated with competitive processes that occur during selection, helping to specify what response should be produced. This process, referred to as "competition resolution," results in suppression of the activity of non-

\footnotetext{
Received Aug. 19, 2011; revised Nov. 1, 2011; accepted Nov. 22, 2011

Author contributions: J.D., E.O., and R.I. designed research; J.D., L.L., and S.V. performed research; J.D. and S.V. analyzed data; J.D., L.L., E.O., and R.B.I. wrote the paper.

This work was supported by grants from the National Institutes of Health (NSO40813), the ARC (Actions de Recherche Concertées, Communauté Française de Belgique), the Fonds Spéciaux de Recherche (FSR) of the Université catholique de Louvain, the Fonds de la Recherche Scientifique Médicale (FRSM), and the Fondation Médicale Reine Elisabeth (FMRE). J.D. was supported by the Belgian National Funds for Scientific Research (FRS-FNRS).

Correspondence should be addressed to Prof. Julie Duque, Institute of Neuroscience, Laboratory of Neurophysiology, Université catholique de Louvain, 53, Avenue Mounier, COSY-B1.53.04, B-1200 Brussels, Belgium. E-mail: julie.duque@uclouvain.be.

DOI:10.1523/JNEUROSCI.4299-12.2012

Copyright $\odot 2012$ the authors $\quad 0270-6474 / 12 / 320806-11 \$ 15.00 / 0$
}

selected response representations (van den Wildenberg et al., 2010; Tandonnet et al., 2011). A second inhibitory mechanism, referred to as "impulse control," is directed at the representation of the selected response (Davranche et al., 2007). It is thought to prevent premature response initiation, controlling when a selected response is executed. By using a variety of stimulation procedures, we observed that these two inhibitory processes operate at different levels of the motor pathways, even though they produce similar effects on global measures of corticospinal excitability (Duque and Ivry, 2009; Duque et al., 2010b). Inhibition related to impulse control modulates spinal excitability, allowing cortical preparatory processes to operate without triggering premature movement, whereas competition resolution arises exclusively from cortical interactions (Fig. 1).

The aim of the present study was to examine the contribution of the frontal cortex to these two forms of inhibition. We focused on dorsal premotor cortex (PMd) and the lateral aspect of prefrontal cortex (LPF), two regions implicated in action selection and preparation (Heekeren et al., 2006; Nakayama et al., 2008). We used a novel procedure in which, during a choice reaction time (RT) task, participants received the sequential application of a high-frequency repetitive transcranial magnetic stimulation (rTMS) train over PMd or LPF followed by single pulse transcranial magnetic stimulation (sTMS) over primary motor cortex (M1). sTMS allowed us to assess inhibitory changes in corticospinal excitability of selected or nonselected responses. rTMS was used to produce transient disruption of PMd or LPF during response preparation. 


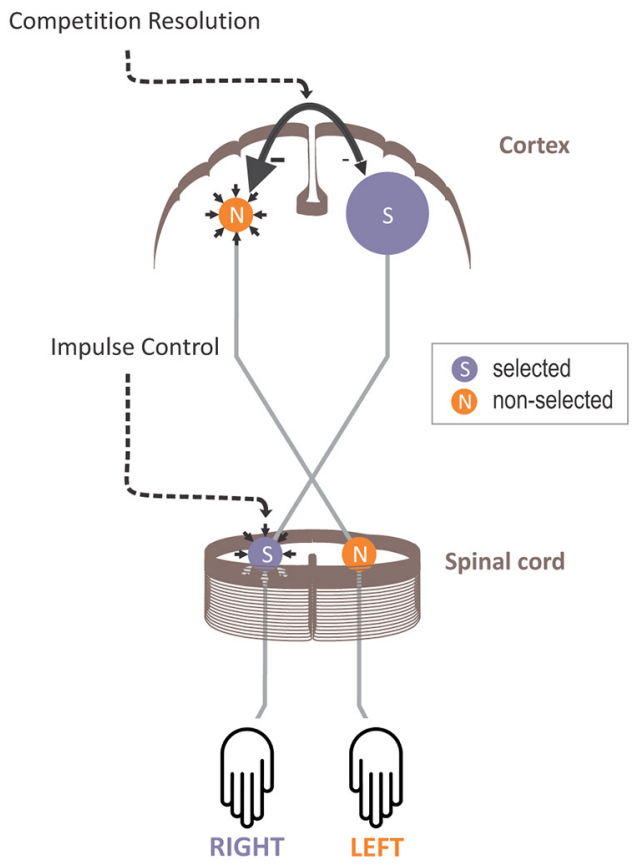

Figure 1. Schematic representation of two mechanisms of inhibition during selection of a right hand response. Inhibition for competition resolution reduces activity of nonselected response representations at the cortical level, possibly through interhemispheric inhibitory interactions. Inhibition for impulse control reduces the activity of selected response representations at the spinal level.

We reasoned that if PMd and/or LPF were the source of one or more of these inhibitory processes, a virtual lesion to these regions would alter this inhibition. Disrupting a brain region involved in competition resolution should reduce inhibition of nonselected responses. We predicted this effect would be observed with rTMS of LPF given the role of this region in goal maintenance, a process that helps ensure that the appropriate response is selected in a variable context (Miller and Cohen, 2001; Koch et al., 2005; Munakata et al., 2011). In contrast, disrupting a region involved in impulse control should reduce inhibition of the selected response. We predicted that this effect would be observed with rTMS of PMd given the role of this region in movement preparation and initiation (Cisek and Kalaska, 2005).

\section{Materials and Methods \\ Participants}

Twelve right-handed healthy subjects $(25 \pm 1.4$ years old; 8 women $)$ participated in this experiment. Handedness was determined via a condensed version of the Edinburgh Handedness Inventory (Oldfield, 1971). Participants were naive to the purpose of the study and financially compensated for their participation. The protocol was approved by the institutional review board at the Universite catholique de Louvain, Brussels, Belgium, and required written informed consent.

\section{Transcranial magnetic stimulation protocol}

We combined sTMS and high-frequency rTMS (10 Hz, $400 \mathrm{~ms}$ ) while subjects performed a choice reaction time task. The sTMS was used to probe corticospinal (CS) excitability during response preparation. To do so, the sTMS coil was positioned over right M1 so as to elicit motorevoked potentials (MEPs) in left first dorsal interosseous (FDI), a muscle required to abduct the index finger. We focused on the left hand because MEP suppression during movement preparation is generally more pronounced in the nondominant hand (Leocani et al., 2000; Duque et al., 2007). The rTMS was used to produce transient virtual lesions in predefined cortical regions. This procedure has been shown to perturb tran- siently the activity in the underlying cortex (i.e., "virtual lesion" method) (Davare et al., 2006; Duque et al., 2010a). In separate sessions on different days, the rTMS coil was placed over the left dorsal premotor cortex (Fig. $2 \mathrm{~A}$, left), the left lateral prefrontal cortex (Fig. $2 \mathrm{~A}$, middle), or the postcentral sulcus (PCs) (Fig. 2A, right). PCs was selected as a control stimulation site.

Because of the size of the transcranial magnetic stimulation (TMS) coils, it was not possible to place the two coils simultaneously over PMd and M1 of the same hemisphere. Thus, our dual-stimulator procedure required that we position the rTMS coil over PMd of the left hemisphere. Importantly, left PMd has been shown to play an important role in response preparation, regardless of whether the left (ipsilateral) or right (contralateral) hand is selected (Schluter et al., 2001; Rushworth et al., 2003). Given this, we reasoned that disruptive effects following rTMS of left PMd should be evident in MEPs elicited in the ipsilateral (left) hand. To maintain consistency, we opted to apply also LPF rTMS over the left hemisphere. However, we also tested a subset of the participants in an additional post hoc session in which the sTMS and LPF rTMS coils were both positioned over the right hemisphere $(n=9 ; 28 \pm 1.5$ years old; 7 women). Note that most subjects recruited in this post hoc session were also part of the pool tested in the main experiment $(n=7)$.

\section{Single pulse TMS}

For the single pulse TMS (sTMS), a $70 \mathrm{~mm}$ figure-of-eight coil connected to a Magstim 200 magnetic stimulator (Magstim) was placed tangentially on the scalp. The handle was oriented toward the back of the head and laterally at a $45^{\circ}$ angle away from the midline, approximately perpendicular to the central sulcus (see Fig. $2 \mathrm{~A}$ ). After fitting the participant with an electroencephalography (EEG) cap, we identified the optimal location for eliciting MEPs in the left FDI. This location was marked on the EEG cap to provide a reference point throughout the experimental session. The resting motor threshold (rMT) was defined as the minimal TMS intensity required to evoke MEPs of about $50 \mu \mathrm{V}$ peak-to-peak in the left FDI on 5 of 10 consecutive trials. As a function of maximum stimulator output, the mean rMTs were $38 \%$ [SE $=2.1$ ], 37\% [SE $=2.3$ ], and $37 \%$ $[\mathrm{SE}=1.9]$ in the left PMd, left LPF, and PCs sessions, respectively. The rMT was $39 \%$ [SE $=1.4]$ in the post hoc right LPF session. The intensity of the sTMS probe was always set at $15 \%$ above the rMT in all sessions.

\section{Repetitive TMS}

Trains of repetitive TMS were applied over PMd and LPF using a second $70 \mathrm{~mm}$ figure-of-eight coil connected to a rapid Magstim 200 magnetic stimulator (Magstim). Each rTMS train involved 5 pulses at $10 \mathrm{~Hz}(400$ ms train duration). The intensity of the rTMS pulses was set at $32 \%$ above the rMT measured with the sTMS coil. Note that there is a loss of power when a coil is powered by a rapid (biphasic pulse) stimulator [see http:// www.magstim.com/magneticstimulators/10108.html]. To account for this effect, the original sTMS rMT was increased by $20 \%$ and the intensity of the rTMS pulses was then set at 10\% above this adjusted rMT value.

Trains of rTMS over LPF can be uncomfortable due to the activation of facial muscles. For this reason, we only recruited participants with a low rMT ( $<50 \%$ of stimulator output). However, when measured in the LPF session, the rMT for one participant was above this criterion (51\%). Given our criterion, this participant was not tested in the LPF session $(n=11)$.

Individual anatomical MRI images were used to guide the placement of the rTMS coil (Noirhomme et al., 2004). Using customized software, we normalized, a posteriori, individual coordinates for the rTMS sites with respect to the MNI brain atlas. This software uses an iterative algorithm that searches for the optimal projection of a given brain onto the MNI brain.

The mean MNI coordinates for the stimulation sites are given in Figure $2 B$. The stimulation site for the left PMd was based on a location used in a previous rTMS study (MNI coordinates: $x=-22, y=-4, z=71$ $\mathrm{mm}$ ) (Davare et al., 2006). This location is just anterior to the precentral sulcus and adjacent to the dorsal bank of the superior frontal sulcus. For the left LPF, we used the average coordinates of three functional neuroimaging studies (Dreher and Berman, 2002; Crone et al., 2006; Schumacher et al., 2007) that reported LPF activation during action selection (MNI 
coordinates: $x=-35, y=25, z=29 \mathrm{~mm}$ ). This stimulation site was adjusted in each individual such that the coil was centered just above the inferior frontal sulcus. The mean MNI coordinates of the actual LPF stimulation site are shown on Figure $2 B$ and correspond to the ventral portion of area BA 9 (Rajkowska and Goldman-Rakic, 1995). The homologous region in the right hemisphere was tested in an additional session in which the coil was positioned over right LPF. For the control site, we opted to stimulate a midline region located just behind the postcentral sulcus, also based on the MRI of each individual subject.

\section{Control experiments}

The protocol used in the task of the main experiment combines two TMS procedures: high-frequency, on-line rTMS to perturb activity in the targeted cortical region (e.g., Davare et al., 2006), and sTMS to probe excitability in the CS pathway (e.g., Leocani et al., 2000). While each of these procedures has been employed in many TMS studies individually, this is the first study in which they are used simultaneously. Given this, we performed several control sessions to examine the effects of high-frequency rTMS on CS excitability when the participants were at rest.

Control Session 1: effect of rTMS over M1 on corticospinal excitability at rest. Repetitive TMS has been used in many studies to induce virtual lesions, perturbing activity in the targeted cortical region (Pascual-Leone et al., 2000; Taylor et al., 2007; Johnson et al., 2010). The disruptive nature of rTMS has generally been inferred by changes in behavior (e.g., Davare et al., 2006). Here, we conducted a control study to assess physiological changes induced by a highfrequency rTMS train on the targeted brain region. TMS methods do not allow a direct assessment of physiological changes in PMd and LPF. However, this is possible if the rTMS train is directed at M1. Thus, in this first control session, we applied rTMS over M1 and measured the amplitude of MEPs elicited by a singlepulse TMS probe (test stimulus) at several delays (from 50 to $2000 \mathrm{~ms}$ ) following the last pulse of the rTMS train (conditioning stimulus). We assume that the pattern of changes evidenced with rTMS to M1 are representative, to a first approximation, of physiological changes that would occur following rTMS to other cortical areas, including PMd and LPF (Allen et al., 2007).

Eight participants ( $30 \pm 2.2$ years old, 6 women) were tested. A single coil $(70 \mathrm{~mm})$ connected to a rapid Magstim 200 magnetic stimulator was used to apply the rTMS pulses and the sTMS probe to right M1. Similar to the procedure in the main experiment, we first identified the hot spot over right M1 for eliciting MEPs in left FDI, and the rMT was defined as the intensity required to evoke MEPs with sTMS on 5 of 10 consecutive trials. The mean $\mathrm{rMT}$ equaled $54 \%$ [SE $=2.7]$. The intensity of TMS for the experimental session was set at $10 \%$ above the rMT. This stimulation level was fixed for both the rTMS and sTMS pulses; thus, MEPs were elicited in response to (most of) the rTMS pulses.

During the experimental session, participants were asked to remain relaxed with the eyes open, the arms semiflexed, and the hands resting, palms down, on a pillow. On rTMS trials, the train $(10 \mathrm{~Hz}, 400 \mathrm{~ms})$ was used as a conditioning stimulus, followed by a sTMS probe (test stimulus). There were 12 different delays between the last pulse of the rTMS train and the sTMS probe: 50, 60, 70, 80, 90, 100, 200, 300, 400, 500, 1000, and $2000 \mathrm{~ms}$. Each interval occurred four times in a test block and each subject was tested on two blocks (eight MEPs total per interval). We also included four catch trials in each block in which the rTMS train was not followed by an sTMS probe. The MEPs evoked by the first pulse of the rTMS trains were used to establish a baseline (MEPs elicited in the absence of a conditioning stimulus).

We also ran two blocks in which the rTMS train was replaced by a single TMS pulse. Thus, in these blocks, two sTMS pulses were applied over M1, separated by 1 of 12 possible delays $(50,60,70,80,90,100,200$, $300,400,500,1000$, and $2000 \mathrm{~ms}$ ). Here, we considered the first sTMS pulse as the conditioning stimulus and the second pulse as the test stimulus. Similar to the rTMS blocks, the MEPs evoked by the conditioning pulse were used to establish a baseline of CS excitability in the absence of a conditioning stimulus.

Repeated measures ANOVA $\left(\mathrm{ANOVA}_{\mathrm{RM}}\right)$ revealed a significant effect of delay on the amplitude of MEPs elicited by the test probe in the rTMS blocks $\left(F_{(12,84)}=7.0, p<0.0001\right.$; Fig. $3 A$, left $)$. MEPs elicited by the test pulse were larger for the $50 \mathrm{~ms}$ delay condition $(p<0.01)$ relative to baseline (MEPs evoked by the first pulse of the rTMS train), followed by an extended period of MEP suppression. This suppression was reliable for delays between 60 and $500 \mathrm{~ms}$ (all $p<0.03$ ). A similar pattern was observed when the conditioning stimulus was a single TMS pulse instead of an rTMS train $\left(F_{(12,84)}=3.8, p<0.0002\right.$; Fig. $3 A$, right). At $50 \mathrm{~ms}$ delay, there was a nonsignificant increase in MEP amplitude ( $p=0.14$ ), followed by a rapid suppression of the MEPs. The suppression did not last as long after the single TMS pulse compared to the rTMS train, remaining significant only for delays between 60 and $100 \mathrm{~ms}$ (all $p<0.05)$. 

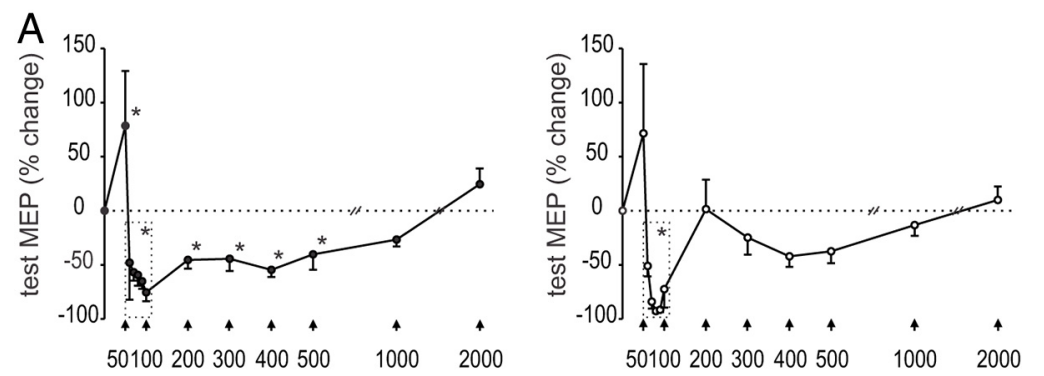

conditioning - test stimulus interval (ms)
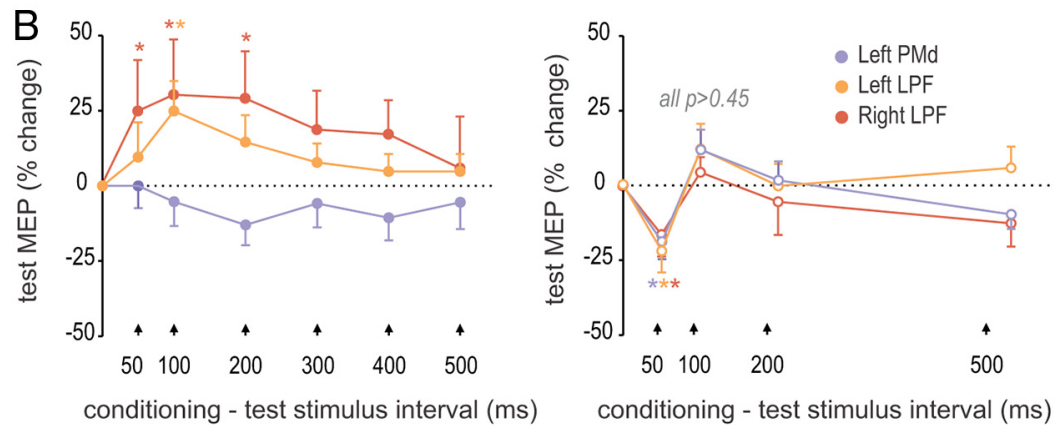

Figure 3. $\boldsymbol{A}$, Control Session 1. MEPs elicited by test sTMS stimulation over right M1 following conditioning stimulation consisting of either right M1 rTMS (left) or sTMS (right). MEPs are expressed with respect to MEPs evoked by the first conditioning pulse. The $\mathrm{x}$-axis indicates the interval between the conditioning and test stimuli. An early facilitatory effect is rapidly followed by inhibition, with the duration of the inhibition much longer following rTMS than sTMS. B, Control Session 2. Effect on MEPs elicited by the test stimulus when the conditioning stimulus is applied over left PMd, left LPF, or right LPF. Conditioned MEPs are expressed with respect to MEPs evoked in the absence of conditioning stimulation. While the three locations produce similar effects with a single pulse conditioning stimulus (right), rTMS of LPF enhances the MEPs independent of the side of stimulation while rTMS of left PMd tends to suppress the MEPs (left) $;^{*} p<0.05$.

These results show that when suprathreshold TMS pulses are applied successively over M1, there is a brief increase in excitability when the delay between two successive pulses is $50 \mathrm{~ms}$, followed by a period of suppression. With an rTMS train of $400 \mathrm{~ms}$ duration, the suppression remains pronounced for up to $500 \mathrm{~ms}$. In the main experiment, we opted to use a delay of $100 \mathrm{~ms}$ between the rTMS train and sTMS probe, given that the suppression of the MEPs was maximal at $100 \mathrm{~ms}$ in this control experiment.

The rapid shift from enhancement to suppression is surprising (note the change from the $50 \mathrm{~ms}$ to the $60 \mathrm{~ms}$ delay conditions) but was observed when either the conditioning stimulus was composed of an rTMS train or a single TMS pulse. The inhibitory effects of the single conditioning pulse is consistent with previous paired-pulse TMS protocols (Nakamura et al., 1997). Studies using long trains of rTMS have revealed a mixture of changes ranging from inhibition to facilitation depending on stimulation intensity and frequency. In brief, low-frequency rTMS $(<5$ $\mathrm{Hz}$ ) generally reduces the excitability of the stimulated neurons (Touge et al., 2001), whereas high-frequency rTMS $(>5 \mathrm{~Hz}$ ) has been reported to produce facilitatory changes (Pascual-Leone et al., 1994; Maeda et al., 2000; Peinemann et al., 2004). None of these studies have looked at the physiological changes that occur immediately $(<1 \mathrm{~s})$ following rTMS, nor with the parameters typically employed in cognitive neuroscience studies designed to induce virtual lesions (Davare et al., 2007; Duque et al., 2010a). Our findings suggest that, in addition to introducing noise to the stimulated region, short high-frequency rTMS trains also reduce cortical excitability for a duration that outlasts the end of the train by 500 ms. This extended suppression of excitability is similar to that observed in a study in which cellular activity was measured in the visual cortex of cats (Allen et al., 2007).

Control Session 2: effect of rTMS over PMd or LPF on corticospinal excitability at rest. In the second control session, the rTMS conditioning stimulus was applied over left PMd $(n=17)$, left LPF $(n=17)$, or right LPF $(n=13)$, followed by a single test stimulus over right M1. The three different rTMS sites were tested in three separate sessions on different days. A total of 19 subjects participated in at least one of these sessions ( $27 \pm 0.9$ years old, 13 women). In all sessions, we combined highfrequency rTMS over one of the regions of interest with sTMS over M1 using a double coil design. This is the same procedure as that used in the main experiment (see Fig. 2), but here the participants were at rest. If one or more of these regions provides inhibitory input to M1 at rest, then perturbing activity in these frontal locations should produce an increase in the MEPs elicited by sTMS of M1. In contrast, if any of these regions provides an excitatory input to right M1, then the rTMS train should produce a decrease in MEPs elicited by sTMS of M1. We recognize that a particular site may provide both inhibitory and excitatory inputs to M1, the strength of which is likely to depend on the functional status. Nonetheless, our aim here was to clarify the effects of a premotor or prefrontal rTMS train on M1 excitability at rest.

CS excitability was assessed by recording MEPs in the left FDI in response to the sTMS probe (test stimulus) applied over the right M1. The mean rMTs equaled $41 \%$ [ $\mathrm{SE}=1.2$ ], $41 \%[\mathrm{SE}=1.2]$, and $37 \%[\mathrm{SE}=1.0]$ in the left PMd, left LPF, and right LPF sessions, respectively. The intensity of the rTMS train was set at $10 \%$ above rMT in all sessions (adjusted to account for the use of different stimulators; see Repetitive TMS); the intensity of the sTMS probe was set at $20 \%$ above rMT. Note that this differs from Control Session 1, where the intensity of the sTMS probe had to be the same as the rTMS train ( $10 \%$ above rMT) since all of the pulses were sent through the same coil over M1. Each rTMS train involved 5 pulses at $10 \mathrm{~Hz}(400 \mathrm{~ms})$. The placement location for PMd and LPF was identical to that used in the main experiment, based on individual anatomical MRIs.

In each session, we tested one region of interest (left PMd, left LPF, or right LPF) and two block types, one in which the test sTMS probe was preceded by a conditioning rTMS train and one in which the test probe was preceded by a conditioning sTMS pulse. Left PMd and left LPF were always tested first, with the order counterbalanced across participants. The right LPF was added a posteriori to assess changes in M1 excitability following rTMS of LPF in the same (right) hemisphere. It was not possible to test the effect of rTMS over right PMd given the size of the coils.

Participants were asked to remain as relaxed as possible with the eyes open, the arms semiflexed, and the hands resting, palms down, on a pillow. In the rTMS blocks, the conditioning rTMS train was followed by the right M1 sTMS probe after one of six possible delays: 50, 100, 200, 300,400 , and $500 \mathrm{~ms}$ (rTMS on trials). The smaller range of delays was selected based on the findings of Control Session 1. Each condition occurred seven times per block, and participants completed three blocks. Each block also included seven trials in which the right M1 sTMS probe was applied alone (rTMS off trials); the mean MEP evoked on these trials was set as the baseline. To assess the effect of the rTMS train, we compared MEPs elicited in the $\mathrm{rTMS}_{\text {on }}$ trials for each delay with respect to baseline ( rTMS $_{\text {off }}$ trials).

We found that repetitive TMS over left $\operatorname{LPF}\left(F_{(6,96)}=2.33, p=0.04\right)$ and right $\operatorname{LPF}\left(F_{(6,72)}=2.66, p=0.02\right)$, significantly altered CS excitability of left FDI at rest (Fig. 3B, left). Perturbation of left LPF increased MEPs in left FDI when the right M1 pulse occurred $100 \mathrm{~ms}$ after the last pulse of the rTMS train (all $p<0.03$ ). A similar increase was observed following perturbation of right LPF, with the effect being statistically reliable at $50 \mathrm{~ms}$ and lasted until $200 \mathrm{~ms}$ (both $p<0.05$ ). The pattern of results was different for left PMd. Overall, MEPs elicited from right M1 stimulation tended to be lower after rTMS of left PMd. However, this 
effect was not reliable for any of the delays $\left(F_{(6,96)}=0.98, p=0.44\right)$, nor was the effect significant when we pooled the data from the delays between 100 and $400 \mathrm{~ms}$, the interval over which the suppression effect appeared strongest (paired $t_{(16)}=1.15, p=0.27$ ).

Reliable changes in right M1 excitability were also observed when the conditioning stimulus was a single TMS pulse, applied over PMd and LPF (all $F>3.2$, all $p<0.02$ ). However, the effects of the single pulse were markedly different than that observed following rTMS trains. For all three regions, left PMd, left LPF, and right LPF, the amplitude of the MEPs was attenuated with a delay of $50 \mathrm{~ms}$ (all $p<$ 0.04, Fig. 3B, right). By $100 \mathrm{~ms}$ and continuing for delays up to $500 \mathrm{~ms}$, the MEPs returned to baseline levels.

In summary, when the conditioning stimulus is a single pulse, there is a marked attenuation of the MEPs with a $50 \mathrm{~ms}$ delay, presumably reflecting an inhibitory link from LPF and PMd to M1 (Ni et al., 2009). When the conditioning stimulus is an rTMS train, a different pattern is observed, consistent with the hypothesis that the rTMS has induced a functional lesion.

The results of Control Session 2 reveal a striking difference between the effects of rTMS over PMd and LPF on the excitability state of M1 at rest. Repetitive TMS over left PMd tended to reduce MEPs elicited by right M1 stimulation, although this effect never reached statistical significance. In contrast, rTMS over left and right LPFs led to an increase in MEPs, an effect that was consistently reliable at $100 \mathrm{~ms}$ for both locations. At first blush, one may be tempted to assume that rTMS of LPF provided an excitatory input to right M1. However, the first control session (where rTMS was applied over M1) suggests that an rTMS train depresses activity in the stimulated area, an assumption critical to studies in which rTMS is used to induce virtual lesions. If we extend the argument to the current experiment, the MEP enhancement would suggest the attenuation of an inhibitory signal from LPF to M1 at rest, or more precisely, during an instruction-based resting period. Given that M1 excitability was reduced after a single TMS pulse, we infer that the projection from LPF includes a significant inhibitory component $(\mathrm{Ni}$ et al., 2009). When rTMS is applied to this region, we assume the functionality is disturbed - that is, we have created a virtual lesion. Disruption of this inhibitory input leads to an increase in M1 excitability, even at rest. We note that "at rest" is a form of an instruction; LPF may be involved in implementing this instruction by inhibiting M1 (MacDonald et al., 2000; Bunge, 2004).

Interestingly, rTMS (and sTMS) of right and left LPFs induced similar patterns of change in right M1. This observation suggests that the functional recruitment of LPF to competition resolution may not be strongly lateralized, an issue we will return to in the Discussion.

The picture is more complex for PMd. As with LPF, a single TMS pulse to left PMd produced an immediate reduction in MEPs elicited from right M1. This suggests that neurons from left PMd have the potential to inhibit right M1 (Ni et al., 2009). More puzzling is the absence of significant modulation of the MEPs following rTMS over PMd, the conditions that, we assume, produce a virtual lesion. One possibility is that PMd does not functionally influence M1 excitability at rest (at least interhemispherically), but rather the influence of PMd on M1 only becomes relevant when a movement has to be performed (Koch et al., 2006; Kroeger et al., 2010).
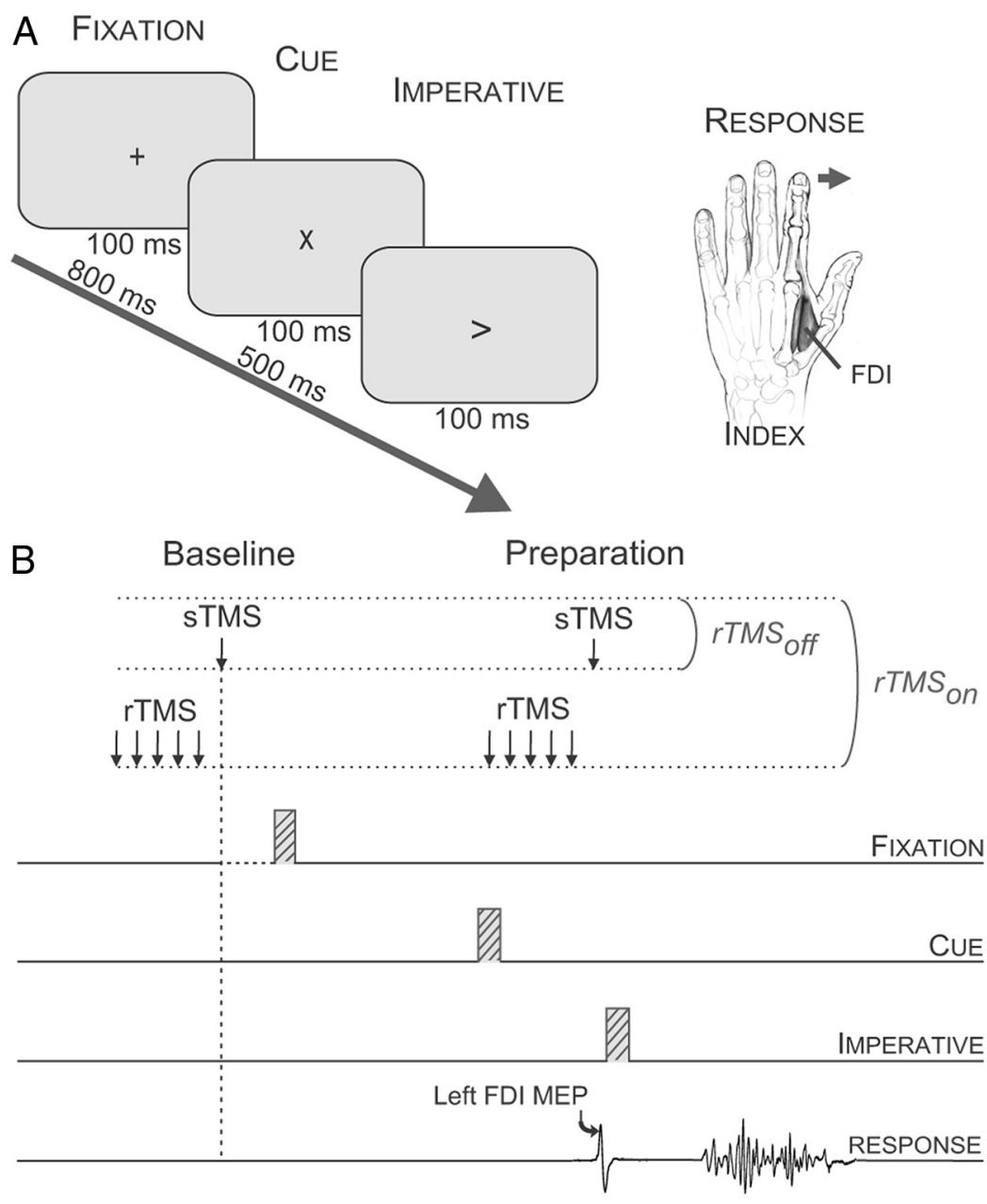

Figure 4. $\quad \boldsymbol{A}$, Illustration of a trial requiring a left index finger response. $\boldsymbol{B}$, A single TMS pulse was applied over the right $\mathrm{M} 1$ at two possible timings (baseline or preparation). This pulse was preceded by an rTMS train on half of the trials (rTMS on trials). On the other half of the trials, the sTMS pulse was presented alone (rTMS ${ }_{\text {off }}$ trials).

\section{Choice reaction time task}

Participants sat in front of a computer screen with both hands resting on a pillow, palms down, with the arms semiflexed. We used a partial cueing task, similar to that in Duque et al. (2010b). A preparatory cue indicated the response hand (left or right), and an imperative signal indicated which of two fingers, index finger or pinky, was to be used on each trial. The preparatory cue was always valid, and participants were instructed to use this information to reduce their RTs.

A fixation cross was presented for $100 \mathrm{~ms}$ to signal the start of each trial (Fig. 4A). After a delay of $800 \mathrm{~ms}$, a preparatory cue was presented for 100 ms. The cue was either the letter " $x$," indicating a forthcoming left hand movement, or the letter, "o," indicating a forthcoming right hand movement. Following the offset of the cue, the screen was blanked for a delay interval of $500 \mathrm{~ms}$ (total delay including the $100 \mathrm{~ms}$ preparatory cue $=$ $600 \mathrm{~ms}$ ). An imperative signal then appeared for $100 \mathrm{~ms}$. The imperative was either a " $<$ " or a " $>$." If the left hand had been cued, these symbols corresponded to pinky and index fingers, respectively; if the right hand had been cued, these symbols corresponded to index and pinky fingers, respectively. The participant was instructed to perform the specified finger movement as quickly as possible following the imperative signal. Following the response, there was an intertrial interval of $5000-5500 \mathrm{~ms}$.

In each session, participants completed eight blocks of 28 trials, each lasting approximately $5 \mathrm{~min}$. The 28 trials consisted of 14 left hand trials and 14 right hand trials, with half of the trials requiring index finger movements, and the other half requiring pinky movements. The first block was used to determine the participant's mean RT in the absence of TMS. RT was defined as the interval between the onset of the imperative 
signal and a movement-related increase in the EMG activity of the agonist muscle [FDI for the index finger response and abductor digiti minimi (ADM) for the pinky finger].

TMS was applied during the other seven blocks. A single pulse was delivered over right M1 on 24 of 28 trials to measure MEPs in the left FDI at one of two timings (Fig. $4 \mathrm{~B}$ ). The first timing was set to obtain a baseline measurement of CS excitability. To this purpose, the sTMS probe was applied at random during the intertrial interval $(200-700 \mathrm{~ms}$ before fixation cross onset) on 8 trials of each block $\left(\mathrm{TMS}_{\text {baseline, }}\right.$ total of 8 trials $\times 7$ blocks $=56$ trials). On the other 16 TMS trials of each block, left MEPs were elicited $550 \mathrm{~ms}$ after the onset of the cue (i.e., $50 \mathrm{~ms}$ before the onset of the imperative signal). At this point, the participant knows the response hand, but the finger remains unknown. Hence, with this second timing we assessed changes in CS excitability associated with response preparation in a selected (left MEPs when left hand cued) or nonselected hand (left MEPs when right hand cued) late in the delay period $\left[\mathrm{TMS}_{\text {preparation, }}\right.$ total of 16 trials ( 8 for each cued hand) $\times 7$ blocks $=112$ trials $=56$ trials per cued hand]. Left MEPs should be suppressed both when the left hand is selected and nonselected, presumably due to the concurrent operation of inhibitory mechanisms related to impulse control and competition resolution, respectively (Duque et al., 2010b). In four catch trials in each block, no sTMS pulse was applied.

Half of the M1 sTMS pulses at TMS baseline $_{\text {and TMS }}$ preparation were preceded by a $400 \mathrm{~ms}$ rTMS train targeted, in separate sessions on different days, either over PMd, LPF, or PCs (or over right LPF in the fourth additional post hoc session). The rTMS train was always fixed to terminate $100 \mathrm{~ms}$ before the sTMS probe (see Fig. $4 \mathrm{~B}$ ). For the TMS ${ }_{\text {baseline }}$ condition, the rTMS train began during the intertrial interval. For the $\mathrm{TMS}_{\text {preparation }}$ condition, the rTMS train began $50 \mathrm{~ms}$ after the cue onset and continued through the delay period, terminating $150 \mathrm{~ms}$ before the imperative signal. The fact that the rTMS train was not applied on half of the M1 sTMS trials allowed us to compare MEP amplitudes on trials with rTMS (rTMS on $_{\text {trials) and without rTMS (rTMS }}$ trials). Moreover, by having both $\mathrm{TMS}_{\text {baseline }}$ and $\mathrm{TMS}_{\text {preparation }}$ probes, we could evaluate the specific effects of the rTMS train during response preparation (e.g., the $\mathrm{TMS}_{\text {preparation }}$ probe). With the current design, we obtained 28 measures of left MEP amplitudes for each of the six experimental conditions $\left[\mathrm{TMS}_{\text {baseline }}\right.$ probe $\left(\mathrm{rTMS}_{\text {on }}\right.$ or $\left.\mathrm{rTMS}_{\text {off }}\right), \mathrm{TMS}_{\text {preparation }}$ with left hand cued $\left(\mathrm{rTMS}_{\text {on }}\right.$ or $\left.\mathrm{rTMS}_{\text {off }}\right)$, or $\mathrm{TMS}_{\text {preparation }}$ with right hand cued $\left(\right.$ rTMS $_{\text {on }}$ or rTMS $\left.\left._{\text {off }}\right)\right]$ within a session.

\section{EMG recording}

EMG activity was recorded from surface electrodes placed over the left and right FDI and ADM muscles. EMG data were collected so that the artifacts of the rTMS and sTMS pulses as well as the motor response were visible on each sweep. The EMG signals were amplified and bandpass filtered on-line (10-500 Hz; Neurolog; Digitimer) and digitized at $2 \mathrm{kHz}$ for off-line analysis. The EMG signals were used to determine the reaction times and measure the peak-to-peak MEP amplitude in left FDI. ADM MEPs were inconsistent and relatively weak, presumably because the hotspot was always selected based on FDI responses; as such, we do not report the ADM data. Trials with background EMG activity $>100 \mu \mathrm{V}$ in the $200 \mathrm{~ms}$ window preceding the TMS pulse were excluded from the analysis. This was done to prevent contamination of the MEP measurements by fluctuations in background EMG (Duque et al., 2005, 2007; Duque and Ivry, 2009). After trimming the data for errors and background EMG activity, a minimum of 20 MEPs remained to assess CS excitability in each condition.

\section{Statistical analysis}

We first analyzed MEPs in the absence of rTMS (rTMS ${ }_{\text {off }}$ trials) to confirm the presence of CS suppression at $\mathrm{TMS}_{\text {preparation }}$ with respect to $\mathrm{TMS}_{\text {baseline. }}$ To do so, we used a series of one-way ANOVAs $\mathrm{RM}_{\text {, }}$, one for each session (PMd, LPF, PCs), with the factor condition $\left(\mathrm{TMS}_{\text {baseline, }}\right.$,

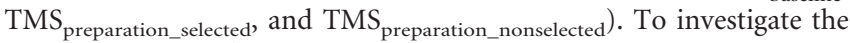
effect of the rTMS "virtual lesions," left FDI MEPs in the rTMS on $_{\text {trials }}$ were expressed with respect to the corresponding MEPs recorded in the rTMS off $_{\text {trials }}\left(\left[\mathrm{rTMS}_{\text {on }}\right]-\left[\mathrm{rTMS}_{\text {off }}\right] /\left[\mathrm{rTMS}_{\text {off }}\right]\right)$. This normal- ized score was then used in a similar series of one-way ANOVAs $s_{\mathrm{RM}}$ (again, one for each session).

RTs were calculated separately for rTMS $_{\text {off }}$ and rTMS $_{\text {on }}$ trials (excluding the "catch" trials). For these analyses, we pooled the data for the index and pinky trials to increase the number of observations. We first analyzed the RT data in the rTMS ${ }_{\text {off }}$ trials by using a series of two-way ANOVA ${ }_{\mathrm{RM}}$ with condition $\left(\mathrm{TMS}_{\text {baseline, }} \mathrm{TMS}_{\text {preparation }}\right)$, and hand (left, right) as factors for each session (PMd, LPF, PCs). Similar to the MEP analysis, RTs in the rTMS on trials were expressed with respect to the corresponding RTs gathered in the $\mathrm{rTMS}_{\text {off }}$ trials $\left(\left[\mathrm{rTMS}_{\text {on }}\right]-\left[\mathrm{rTMS}_{\text {off }}\right] /\right.$ $\left.\left[\mathrm{rTMS}_{\text {off }}\right]\right)$. This normalized score was then used in a series of two-way ANOVAs $_{\mathrm{RM}}$.

All post hoc comparisons were conducted using the Fisher's Least Significant Difference procedure. Single sample $t$ tests were used to assess the significance of rTMS-related changes with respect to the 0 value (no change). All of the data are expressed as mean \pm SE.

\section{Results}

We observed the same effects for right and left LPF stimulation. For the sake of clarity, we only report the results from left LPF stimulation in the main paper, providing a comparison of the effects of rTMS of LPF and PMd for the same (left) hemisphere.

\section{MEP measurements}

Figure $5 A$ shows the mean MEP amplitudes in the absence of rTMS (rTMS ${ }_{\text {off }}$ trials). The data are plotted separately for the PMd and LPF sites, as well as for our control site, PCs. MEPs elicited during the intertrial interval $\left(\mathrm{TMS}_{\text {baseline }}\right)$ equaled $1.1 \mathrm{mV}[\mathrm{SE}=0.19, n=12], 1.6 \mathrm{mV}$ $[\mathrm{SE}=0.26, n=11]$ and $1.1 \mathrm{mV}[\mathrm{SE}=0.18, n=12]$ in the PMd, LPF, and PCs sessions, respectively. We assume that these differences are indicative of the variation classically observed in MEP measurements across different sessions. The MEPs were strongly suppressed at TM$\mathrm{S}_{\text {preparation }}$ elicited at the end of the delay period (main effect of condition: $F_{(2,22)}=6.3, p<0.007 ; F_{(2,20)}=14.9, p<0.0001$; and $F_{(2,22)}=9.3$, $p<0.002$ in the PMd, LPF and PCs sessions, respectively; Fig. $5 A$ ). This suppression was evident both on trials in which the left hand was selected for the forthcoming response (TMS average suppression $=36 \%$, all three sessions $p<0.003$ ) and when the left hand was not selected for the forthcoming response (right hand response; $\mathrm{TMS}_{\text {preparation_nonselected }}$, average $=28 \%$, all $p<0.02$ ). In our previous studies (Duque and Ivry, 2009; Duque et al., 2010b), we reported that left MEP suppression was systematically larger when the left hand was selected. This pattern was also evident here, although the difference between trials in which the left hand was selected and nonselected was not significant $(p=0.08)$. Based on other stimulation protocols (Duque and Ivry, 2009; Duque et al., 2010b), we assumed that the delay period suppression of the left MEPs reflects the operation of two distinct inhibitory processes: impulse control when the left hand is selected and competition resolution when the right hand is selected (left hand nonselected). Note that part of the left MEP suppression in the left response condition might also reflect inhibition for competition resolution, explaining why the MEP suppression is consistently larger in a selected hand (competition resolution and impulse control) compared to a nonselected hand (competition resolution only).

The effects of rTMS-induced virtual lesions of PMd, LPF, and PCs are shown in Figure 5B. For each panel, the data are presented as a change in MEP amplitude following rTMS $\left(\mathrm{rTMS}_{\text {on }}\right.$ trials) relative to the MEP amplitude when rTMS was absent (rTMS $_{\text {off }}$ trials). The data from the control stimulation site, PCs, indicate that cortical rTMS does not produce a nonspecific attenuation of MEP suppression during response preparation. Repetitive TMS over PCs had no effect on MEPs elicited by the sTMS 
M1 probe (Fig. 5B, right). There was a trend for PCs rTMS to produce smaller MEPs at baseline and larger MEPs during the delay period. However, none of these changes were significantly different from zero (all $p$ values $>0.14$ ), nor were there any differences between the three conditions in terms of rTMS-induced changes.

Repetitive TMS over PMd produced a selective change in the amplitude of MEPs elicited by the sTMS M1 probe $\left(F_{(2,22)}=\right.$ 4.3, $p<0.03)$. As shown in Figure $5 B$ (left), rTMS attenuated MEP suppression during the delay period $\left(\mathrm{TMS}_{\text {preparation }}\right)$, but only in trials in which the left hand was selected for the forthcoming response (percentage change larger than 0 ). Left MEPs elicited at $\mathrm{TMS}_{\text {preparation_selected }}$ were $35 \%$ larger in the rTMS $_{\text {on }}$ compared to the $\mathrm{rTMS}_{\text {off }}$ trials. The MEPs were still suppressed in $\mathrm{rTMS}_{\text {on }}$ trials, but the magnitude of this suppression was only $18 \%$ following PMd rTMS, a value that is significantly smaller than the $33 \%$ reduction obtained in the rTMS ${ }_{\text {off }}$ trials $(p=0.03)$. Importantly, there was no change in the magnitude of MEP suppression during the delay period in the (nonselected) left hand when participants prepared a right hand response $\left(\mathrm{TMS}_{\text {preparation_nonselected }} ; p=0.96\right)$, nor was there an effect of PMd rTMS on MEPs elicited at $\mathrm{TMS}_{\text {baseline }}(p=0.26)$. Post hoc paired-comparisons showed that the effect of rTMS of PMd produced a significant difference in the condition in which the left hand was selected compared to when this hand was not selected $(p=0.04)$ or from that induced by rTMS at baseline $(p=0.01)$. These results suggest that PMd is involved in a process producing inhibition on selected representations (impulse control) but does not induce inhibition targeted at nonselected representations (competition resolution).

A different picture emerged when the rTMS train was directed at LPF (Fig. 5B, middle). MEPs elicited by the M1 probe were also significantly changed by rTMS of $\operatorname{LPF}\left(F_{(2,20)}=8.5, p<0.002\right)$. Post hoc tests revealed that here the delay period inhibition was reduced on trials in which the left hand was selected $(p=0.01)$ and on trials in which the left hand was nonselected (right hand selected; $p=0.03$ ). Relative to MEPs elicited at $\mathrm{TMS}_{\text {baseline, }}$ MEPs in the $\mathrm{rTMS}_{\text {on }}$ trials were inhibited by 13 and $12 \%$ on selected and nonselected trials, respectively, values that were significantly lower than those observed when rTMS was absent $\left(\mathrm{rTMS}_{\text {off }}\right.$ trials: 33 and 25\%). These rTMS-induced changes on MEPs elicited during the delay period were significantly different from those observed when the rTMS train was applied at baseline (preceding MEPs elicited at $\mathrm{TMS}_{\text {baseline; }}$ both $p<0.02$ ). Importantly, the effects of LPF rTMS were also significantly different from the effects observed following rTMS of the control postcentral sulcus

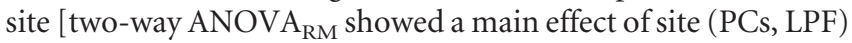
regardless of the selected hand: $\left.F_{(1,10)}=7.78, p<0.02\right]$. These results suggest that LPF inhibits both selected and nonselected response representations during motor preparation, possibly as part of an inhibitory process associated with competition resolution.

In summary, we found that rTMS of both LPF and PMd altered MEP suppression in conditions where the left hand was selected; virtual lesions of each frontal region produced an increase in MEPs (i.e., reduced inhibition) relative to when the rTMS train was absent. In contrast, the left MEP suppression observed in conditions where the right hand was selected (left hand nonselected) was only altered by rTMS over LPF.

\section{Behavioral measurements}

Overall, error rates were $<2 \%$, and this number did not differ between stimulation sites and did not increase on trials with rTMS. Reaction times were quite short, averaging $289 \mathrm{~ms}$ across conditions in which only sTMS was applied. There was no difference in RT between left and right hand responses (main effect of hand: all session, $F<3.5, p>0.1$ ). Given this, the RT data were pooled across the two hands (Fig. 6A). For the TMS $_{\text {off }}$ trials, there was no increase in RT when the sTMS probe was applied in the delay period compared to when it was applied at baseline (main effect of condition: all session, $F<2.5, p>0.1$ ). 
A Reaction times in the absence of rTMS
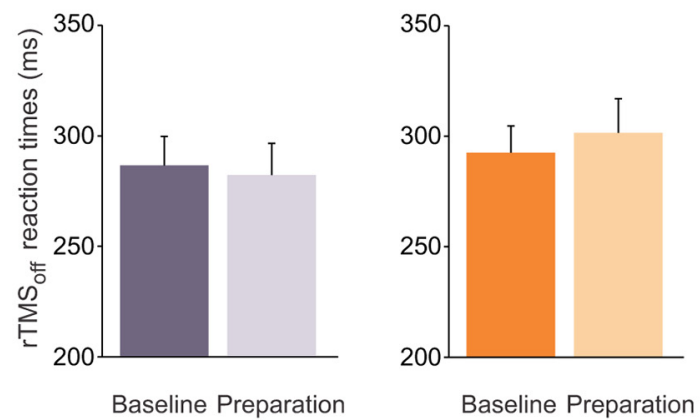

B rTMS induced changes in reaction times

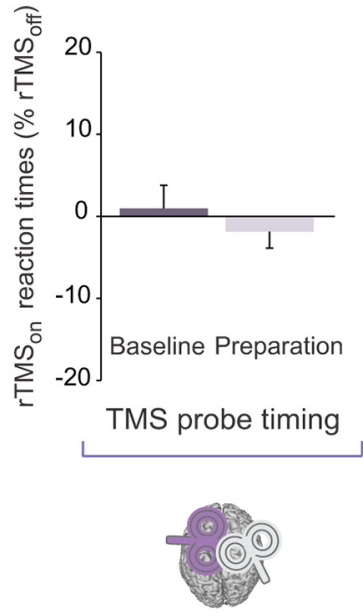

PMd session
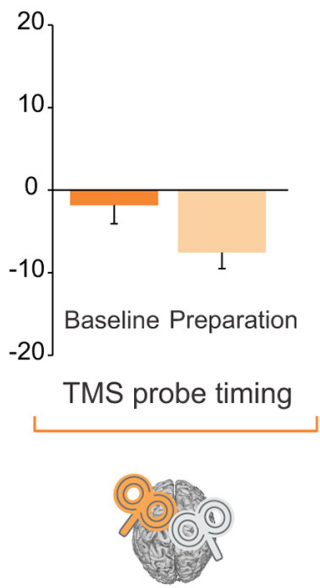

LPF session
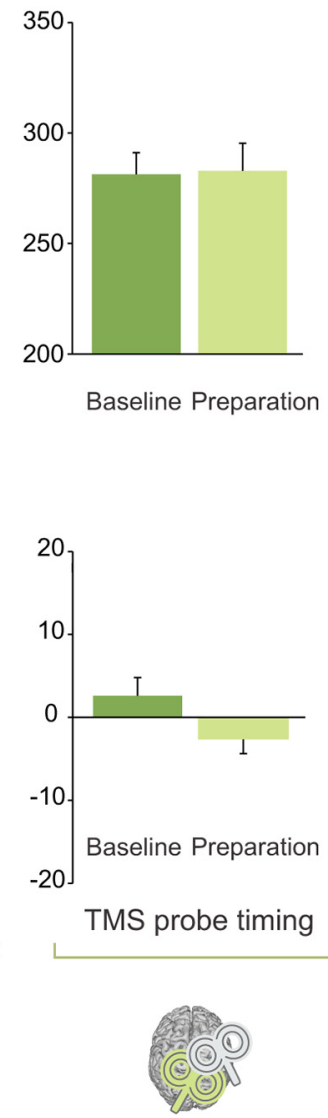

PCs session

Figure 6. A, Reaction times (ms) recorded on rTMS off trials during the PMd (left), LPF (middle), and PCs (right) sessions. Data for

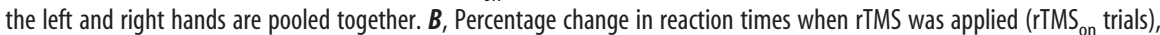
expressed with respect to RTs observed in the absence of rTMS (rTMS ${ }_{\text {off }}$ trials). There was a trend for LPF rTMS to shorten reaction times when applied during the delay preparation period.

The inclusion of an rTMS train during the delay period also had a negligible effect on RTs. Figure $6 B$ plots these data as a function of the change in $\mathrm{rTMS}_{\text {on }}$ trials with respect to the RTs in $\mathrm{rTMS}_{\text {off }}$ trials. The only effect that approached significance in the ANOVAs was when $r T M S$ was applied over the $\operatorname{LPF}\left(F_{(1,10)}=4.2\right.$, $p=0.07)$. The trend here was for shorter RTs when the rTMS train was applied during the delay period compared to trials in which rTMS occurred during baseline. The decrease in RTs following rTMS over LPF during the delay period was significantly different than zero, an effect observed on both left and right hand trials, pooled together in Figure $6 B$ (both $p<0.05$ ). Such a change was not observed following right LPF stimulation $\left(F_{(1,8)}\right.$ $=0.4, p=0.5)$, although the data also show a trend for shorter RTs during the delay period in the rTMS on $_{\text {trials. }}$

\section{Discussion}

Recently we proposed that the inhibitory effects observed in selected and nonselected effectors during motor preparation reflect the operation of two distinct inhibitory mechanisms (Duque et al., 2010b). Here, we provide converging evidence in support of this idea by demonstrating that inhibition of the selected and nonselected effectors were differently affected by rTMS applied over PMd or LPF. Repetitive TMS applied over PMd distinctively attenuated inhibition in the selected effector; in contrast, rTMS over LPF attenuated inhibition in both the selected and nonselected effectors.

\section{PMd and impulse control}

We found that rTMS over PMd specifically attenuated inhibition in a selected effector before the onset of an imperative signal. When the preparatory cue indicated a left hand response, left MEPs were significantly larger (less suppressed) when the M1 sTMS pulse was preceded by an rTMS train over PMd compared to when the sTMS pulse was applied alone. In contrast, left MEPs remained unchanged by rTMS when the preparatory cue indicated a right hand response. Thus, rTMS over PMd did not alter inhibition targeted at a nonselected effector. These findings strongly point to a role of PMd in impulse control.

Faster RTs were not observed in the rTMS $_{\text {on }}$ trials, a result that might be expected if impulse control were disrupted. While caution is required in interpreting null results, we must emphasize that impulse control is not the sole function of PMd but is rather one part of its preparatory activity. It is possible that, in the present study, in addition to disrupting impulse control, rTMS also attenuated activation of the prepared response (Passingham, 1993; Schluter et al., 1998).

PMd plays a critical role in the selection and implementation of action plans, especially in the context of visuomotor associations (Grafton et al., 1998; CavinaPratesi et al., 2006; Hoshi and Tanji, 2006; Terao et al., 2007). When an upcoming action is cued but needs to be withheld until an imperative signal, PMd neurons exhibit tuning for parameters of the selected response during the delay period (Cisek and Kalaska, 2005; Churchland et al., 2006). Inhibition related to impulse control allows this cortical preparation to occur without causing undesired premature movements (Boulinguez et al., 2008; Kaufman et al., 2010). The present findings suggest that PMd contributes to the generation of these inhibitory signals. That is, PMd may not only help to specify the selected movement, but also generate inhibitory signals to block motor output until the movement is initiated (Prut and Fetz, 1999).

The current results are consistent with previous studies pointing to a role of PMd in impulse control. In monkeys, the injection of $\mathrm{GABA}_{\mathrm{A}}$ antagonist within PMd reduces the ability to withhold movements (Sawaguchi et al., 1996). Moreover, using a pairedpulse TMS protocol in which single TMS pulses were applied sequentially over PMd and M1, MEP suppression was observed in the selected effector during a delay period (Kroeger et al., 2010). Intriguingly, when a similar TMS protocol is applied after the onset of the imperative, the effect is reversed with inhibition now observed in nonselected effectors (Koch et al., 2006; but see also O'Shea et al., 2007).

Electrophysiological studies in humans and monkeys have revealed that the excitability of agonist motoneurons is reduced 
during delay periods (Touge et al., 1998; Hasbroucq et al., 1999; Prut and Fetz, 1999; Kaufman et al., 2010), suggesting that impulse control operates by inhibiting the motor output at the spinal level. This raises the question of how PMd modulates spinal cord excitability. One possibility is through CS projections, since a proportion of the CS tract originates from pyramidal cells located in PMd (Dum and Strick, 1991). Interestingly, whereas M1 cells predominantly terminate on spinal motoneurons controlling distal hand muscles, PMd terminations are primarily directed to spinal interneurons (Galea and Darian-Smith, 1994; Dum and Strick, 2005). Given this anatomical organization, PMd has been associated with the preparation of the spinal motor circuitry (Bizzi et al., 2000) rather than in the generation of finger movements, consistent with our impulse control hypothesis.

Impulse control might also arise via PMd modulation of subcortical regions. There is a prominent PMd projection to basal ganglia (McFarland and Haber, 2002), a region linked to response initiation and inhibition (Aron, 2007). PMd might also influence spinal excitability via the brainstem. There are significant projections from the brainstem to interneurons located in the intermediate zone of the spinal cord (primarily part of the reticulospinal tract) (Riddle et al., 2009) that are involved in the control of distal hand muscles (Borra et al., 2010; Cohen et al., 2010).

The attenuation of impulse control on the selected effector (left FDI) resulted from the application of rTMS over the left ipsilateral PMd. We expect we would have observed similar effects if the rTMS train was directed at contralateral PMd (right hemisphere), although the size of the coils precludes a test of this hypothesis. Nonetheless, it is important to consider how ipsilateral cortex could selectively target the selected response, especially since most PMd CS fibers project to the contralateral spinal cord (Nathan et al., 1990). One possibility is that homologous regions of PMd work in concert via transcallosal fibers to produce impulse control (Marconi et al., 2003; Hofer and Frahm, 2006). Alternatively, left PMd might influence spinal excitability via descending pathways originating from subcortical structures that target ipsilateral spinal interneurons (Riddle and Baker, 2010).

\section{LPF and competition resolution}

In contrast to what was found for PMd, rTMS over LPF reduced inhibition in the representation of the nonselected effector, consistent with the hypothesis that this region is a source of inhibition related to competition resolution (Ridderinkhof et al., 2004; Sumner et al., 2010). Our initial expectation was that disruption of competition resolution would be limited to the nonselected effector. However, repetitive TMS over LPF also affected suppression of the selected effector, similarly to that observed when rTMS was applied over PMd. It is possible that LPF is also a source of signals related to impulse control (Risterucci et al., 2003; Narayanan and Laubach, 2006).

Alternatively, the reduced inhibition for both selected and nonselected conditions may reflect a generic effect of LPF rTMS on competition resolution, one that is manifest on all taskrelevant representations. Such an effect may reflect the operation of a winner-take-all network (Coles et al., 1985; Usher and McClelland, 2004; Brown and Heathcote, 2005), but one in which there are mutual inhibitory links between potential responses (Ferrera and Lisberger, 1995; Sheliga et al., 2006). In the current study, the onset of the preparatory cue would trigger a competition between preparatory processes associated with right and left hand responses, with each preparatory process producing some inhibition of the other alternative. Over time, the dynamics will favor the response indicated by the cue. Nonetheless, representations of the nonselected action will also have produced some inhibition targeted at the selected action. The similar effects of rTMS of LPF on trials in which the left hand is selected and not selected may thus reflect the disruption of mutual inhibitory signals associated with competition resolution.

The preceding argument suggests that during the delay period the selected action is subject to the joint influence of impulse control and competition resolution; the nonselected action is subject only to competition resolution. This is consistent with the observation that MEP suppression is systematically stronger on selected trials than on nonselected trials (Duque and Ivry, 2009). Additionally, there was a trend for faster RTs following rTMS of LPF, independent of whether the responses were made with the left or right hand. This hastening may be the consequence of a reduced inhibition related to competition resolution in the selected hand.

Prefrontal cortex is associated with the implementation of higher-order rules and strategies (Fuster, 2001; Koechlin and Summerfield, 2007). During delayed response tasks, LPF cells show sustained activity reflecting the goal of a forthcoming action in a given context (Fuster, 2000; Rowe et al., 2000; Hester et al., 2007). Our results suggest that, as part of this goal-based function, LPF helps sharpen competitive processes, ensuring that the appropriate actions are selected given information specifying the context (e.g., hand) for the forthcoming trial (Fassbender et al., 2009).

Our prior studies indicate that, unlike the spinal manifestation of impulse control, competition resolution is limited to supraspinal interactions (Duque et al., 2010b). This competition may entail corticocortical dynamics that presumably include transcallosal interactions essential for movement preparation (Geschwind and Kaplan, 1962; Franz et al., 1996; Kennerley et al., 2002). In this context of hand selection, LPF may modulate these interactions by imposing task-constraints on areas, such as supplementary motor area, premotor cortex, or even M1, that are more directly involved in the preparation and implementation of the selected movement (Dum and Strick, 2005). It is also possible that the inhibitory influence of LPF to competition resolution occurs through the basal ganglia (Coulthard et al., 2008), similar to what was proposed in the sensory domain (Brunia, 1999; Knight et al., 1999; Frank et al., 2007; Frank, 2011). The current results underscore that the dynamics underlying the translation of a goal into a movement involve the operation of inhibitory processes.

\section{Notes}

Supplemental material for this article is available at http://www. julieduque.com/publications. Illustration of stimulation sites and results in the right LPF session This material has not been peer reviewed.

\section{References}

Allen EA, Pasley BN, Duong T, Freeman RD (2007) Transcranial magnetic stimulation elicits coupled neural and hemodynamic consequences. Science 317:1918-1921.

Aron AR (2007) The neural basis of inhibition in cognitive control. Neuroscientist 13:214-228.

Bizzi E, Tresch MC, Saltiel P, d'Avella A (2000) New perspectives on spinal motor systems. Nat Rev Neurosci 1:101-108.

Borra E, Belmalih A, Gerbella M, Rozzi S, Luppino G (2010) Projections of the hand field of the macaque ventral premotor area F5 to the brainstem and spinal cord. J Comp Neurol 518:2570-2591.

Boulinguez P, Jaffard M, Granjon L, Benraiss A (2008) Warning signals induce automatic EMG activations and proactive volitional inhibition: ev- 
idence from analysis of error distribution in simple RT. J Neurophysiol 99:1572-1578.

Brown S, Heathcote A (2005) A ballistic model of choice response time. Psychol Rev 112:117-128.

Brunia CH (1999) Neural aspects of anticipatory behavior. Acta Psychol (Amst) 101:213-242.

Bunge SA (2004) How we use rules to select actions: a review of evidence from cognitive neuroscience. Cogn Affect Behav Neurosci 4:564-579.

Cai W, Oldenkamp CL, Aron AR (2011) A proactive mechanism for selective suppression of response tendencies. J Neurosci 31:5965-5969.

Cavina-Pratesi C, Valyear KF, Culham JC, Köhler S, Obhi SS, Marzi CA, Goodale MA (2006) Dissociating arbitrary stimulus-response mapping from movement planning during preparatory period: evidence from event-related functional magnetic resonance imaging. J Neurosci 26:2704-2713.

Churchland MM, Yu BM, Ryu SI, Santhanam G, Shenoy KV (2006) Neural variability in premotor cortex provides a signature of motor preparation. J Neurosci 26:3697-3712.

Cisek P, Kalaska JF (2005) Neural correlates of reaching decisions in dorsal premotor cortex: specification of multiple direction choices and final selection of action. Neuron 45:801-814.

Cisek P, Kalaska JF (2010) Neural mechanisms for interacting with a world full of action choices. Annu Rev Neurosci 33:269-298.

Cohen O, Sherman E, Zinger N, Perlmutter S, Prut Y (2010) Getting ready to move: transmitted information in the corticospinal pathway during preparation for movement. Curr Opin Neurobiol 20:696-703.

Coles MG, Gratton G, Bashore TR, Eriksen CW, Donchin E (1985) A psychophysiological investigation of the continuous flow model of human information processing. J Exp Psychol Hum Percept Perform 11:529-553.

Coulthard E, Rudd A, Husain M (2008) Motor neglect associated with loss of action inhibition. J Neurol Neurosurg Psychiatry 79:1401-1404.

Crone EA, Wendelken C, Donohue SE, Bunge SA (2006) Neural evidence for dissociable components of task-switching. Cereb Cortex 16:475-486.

Davare M, Andres M, Cosnard G, Thonnard JL, Olivier E (2006) Dissociating the role of ventral and dorsal premotor cortex in precision grasping. J Neurosci 26:2260-2268.

Davare M, Andres M, Clerget E, Thonnard JL, Olivier E (2007) Temporal dissociation between hand shaping and grip force scaling in the anterior intraparietal area. J Neurosci 27:3974-3980.

Davranche K, Tandonnet C, Burle B, Meynier C, Vidal F, Hasbroucq T (2007) The dual nature of time preparation: neural activation and suppression revealed by transcranial magnetic stimulation of the motor cortex. Eur J Neurosci 25:3766-3774.

Dreher JC, Berman KF (2002) Fractionating the neural substrate of cognitive control processes. Proc Natl Acad Sci U S A 99:14595-14600.

Dum RP, Strick PL (1991) The origin of corticospinal projections from the premotor areas in the frontal lobe. J Neurosci 11:667-689.

Dum RP, Strick PL (2005) Frontal lobe inputs to the digit representations of the motor areas on the lateral surface of the hemisphere. J Neurosci 25:1375-1386.

Duque J, Ivry RB (2009) Role of corticospinal suppression during motor preparation. Cereb Cortex 19:2013-2024.

Duque J, Mazzocchio R, Dambrosia J, Murase N, Olivier E, Cohen LG (2005) Kinematically specific interhemispheric inhibition operating in the process of generation of a voluntary movement. Cereb Cortex 15:588-593.

Duque J, Murase N, Celnik P, Hummel F, Harris-Love M, Mazzocchio R, Olivier E, Cohen LG (2007) Intermanual Differences in Movementrelated Interhemispheric Inhibition. J Cogn Neurosci 19:204-213.

Duque J, Davare M, Delaunay L, Jacob B, Saur R, Hummel F, Hermoye L, Rossion B, Olivier E (2010a) Monitoring coordination during bimanual movements: where is the mastermind? J Cogn Neurosci 22:526-542.

Duque J, Lew D, Mazzocchio R, Olivier E, Ivry RB (2010b) Evidence for two concurrent inhibitory mechanisms during response preparation. J Neurosci 30:3793-3802.

Fassbender C, Hester R, Murphy K, Foxe JJ, Foxe DM, Garavan H (2009) Prefrontal and midline interactions mediating behavioural control. Eur J Neurosci 29:181-187.

Ferrera VP, Lisberger SG (1995) Attention and target selection for smooth pursuit eye movements. J Neurosci 15:7472-7484.

Frank MJ (2011) Computational models of motivated action selection in corticostriatal circuits. Curr Opin Neurobiol 21:381-386.
Frank MJ, Samanta J, Moustafa AA, Sherman SJ (2007) Hold your horses: impulsivity, deep brain stimulation, and medication in parkinsonism. Science 318:1309-1312.

Franz EA, Eliassen JC, Ivry RB, Gazzaniga MS (1996) Dissociation of spatial and temporal coupling in the bimanual movements of callosotomy patients. Psychol Sci 7:306-310.

Fuster JM (2000) Prefrontal neurons in networks of executive memory. Brain Res Bull 52:331-336.

Fuster JM (2001) The prefrontal cortex-an update: time is of the essence. Neuron 30:319-333.

Galea MP, Darian-Smith I (1994) Multiple corticospinal neuron populations in the macaque monkey are specified by their unique cortical origins, spinal terminations, and connections. Cereb Cortex 4:166-194.

Geschwind N, Kaplan E (1962) A human cerebral deconnection syndrome. A preliminary report. Neurology 12:675-685.

Grafton ST, Fagg AH, Arbib MA (1998) Dorsal premotor cortex and conditional movement selection: A PET functional mapping study. J Neurophysiol 79:1092-1097.

Hasbroucq T, Kaneko H, Akamatsu M, Possamaï CA (1999) The timecourse of preparatory spinal and cortico-spinal inhibition: an H-reflex and transcranial magnetic stimulation study in man. Exp Brain Res 124:33-41.

Heekeren HR, Marrett S, Ruff DA, Bandettini PA, Ungerleider LG (2006) Involvement of human left dorsolateral prefrontal cortex in perceptual decision making is independent of response modality. Proc Natl Acad Sci U S A 103:10023-10028.

Hester R, D'Esposito M, Cole MW, Garavan H (2007) Neural mechanisms for response selection: comparing selection of responses and items from working memory. Neuroimage 34:446-454.

Hofer S, Frahm J (2006) Topography of the human corpus callosum revisited-comprehensive fiber tractography using diffusion tensor magnetic resonance imaging. Neuroimage 32:989-994.

Hoshi E, Tanji J (2006) Differential involvement of neurons in the dorsal and ventral premotor cortex during processing of visual signals for action planning. J Neurophysiol 95:3596-3616.

Johnson JS, Hamidi M, Postle BR (2010) Using EEG to explore how rTMS produces its effects on behavior. Brain Topogr 22:281-293.

Kaufman MT, Churchland MM, Santhanam G, Yu BM, Afshar A, Ryu SI, Shenoy KV (2010) Roles of monkey premotor neuron classes in movement preparation and execution. J Neurophysiol 104:799-810.

Kennerley SW, Diedrichsen J, Hazeltine E, Semjen A, Ivry RB (2002) Callosotomy patients exhibit temporal uncoupling during continuous bimanual movements. Nat Neurosci 5:376-381.

Knight RT, Staines WR, Swick D, Chao LL (1999) Prefrontal cortex regulates inhibition and excitation in distributed neural networks. Acta Psychol (Amst) 101:159-178.

Koch G, Oliveri M, Torriero S, Carlesimo GA, Turriziani P, Caltagirone C (2005) rTMS evidence of different delay and decision processes in a fronto-parietal neuronal network activated during spatial working memory. Neuroimage 24:34-39.

Koch G, Franca M, Del Olmo MF, Cheeran B, Milton R, Alvarez Sauco M, Rothwell JC (2006) Time course of functional connectivity between dorsal premotor and contralateral motor cortex during movement selection. J Neurosci 26:7452-7459.

Koechlin E, Summerfield C (2007) An information theoretical approach to prefrontal executive function. Trends Cogn Sci 11:229-235.

Kroeger J, Bäumer T, Jonas M, Rothwell JC, Siebner HR, Münchau A (2010) Charting the excitability of premotor to motor connections while withholding or initiating a selected movement. Eur J Neurosci 32:1771-1779.

Leocani L, Cohen LG, Wassermann EM, Ikoma K, Hallett M (2000) Human corticospinal excitability evaluated with transcranial magnetic stimulation during different reaction time paradigms. Brain 123:1161-1173.

MacDonald AW 3rd, Cohen JD, Stenger VA, Carter CS (2000) Dissociating the role of the dorsolateral prefrontal and anterior cingulate cortex in cognitive control. Science 288:1835-1838.

Maeda F, Keenan JP, Tormos JM, Topka H, Pascual-Leone A (2000) Modulation of corticospinal excitability by repetitive transcranial magnetic stimulation. Clin Neurophysiol 111:800-805.

Marconi B, Genovesio A, Giannetti S, Molinari M, Caminiti R (2003) Callosal connections of dorso-lateral premotor cortex. Eur J Neurosci 18:775-788.

McFarland NR, Haber SN (2002) Thalamic relay nuclei of the basal ganglia 
form both reciprocal and nonreciprocal cortical connections, linking multiple frontal cortical areas. J Neurosci 22:8117-8132.

Miller EK, Cohen JD (2001) An integrative theory of prefrontal cortex function. Annu Rev Neurosci 24:167-202.

Munakata Y, Herd SA, Chatham CH, Depue BE, Banich MT, O’Reilly RC (2011) A unified framework for inhibitory control. Trends Cogn Sci 15:453-459.

Nakamura H, Kitagawa H, Kawaguchi Y, Tsuji H (1997) Intracortical facilitation and inhibition after transcranial magnetic stimulation in conscious humans. J Physiol 498:817-823.

Nakayama Y, Yamagata T, Tanji J, Hoshi E (2008) Transformation of a virtual action plan into a motor plan in the premotor cortex. J Neurosci 28:10287-10297.

Narayanan NS, Laubach M (2006) Top-down control of motor cortex ensembles by dorsomedial prefrontal cortex. Neuron 52:921-931.

Nathan PW, Smith MC, Deacon P (1990) The corticospinal tracts in man. Course and location of fibres at different segmental levels. Brain 113:303-324.

Ni Z, Gunraj C, Nelson AJ, Yeh IJ, Castillo G, Hoque T, Chen R (2009) Two phases of interhemispheric inhibition between motor related cortical areas and the primary motor cortex in human. Cereb Cortex 19:1654-1665.

Noirhomme Q, Ferrant M, Vandermeeren Y, Olivier E, Macq B, Cuisenaire O (2004) Registration and real-time visualization of transcranial magnetic stimulation with 3-D MR images. IEEE Trans Biomed Eng 51:1994-2005.

Oldfield RC (1971) The assessment and analysis of handedness: the Edinburgh inventory. Neuropsychologia 9:97-113.

O'Shea J, Johansen-Berg H, Trief D, Göbel S, Rushworth MF (2007) Functionally specific reorganization in human premotor cortex. Neuron 54:479-490.

Pascual-Leone A, Valls-Solé J, Wassermann EM, Hallett M (1994) Responses to rapid-rate transcranial magnetic stimulation of the human motor cortex. Brain 117:847-858.

Pascual-Leone A, Walsh V, Rothwell J (2000) Transcranial magnetic stimulation in cognitive neuroscience-virtual lesion, chronometry, and functional connectivity. Curr Opin Neurobiol 10:232-237.

Passingham RE (1993) The frontal lobes and voluntary action. Oxford: Oxford UP.

Peinemann A, Reimer B, Löer C, Quartarone A, Münchau A, Conrad B, Siebner HR (2004) Long-lasting increase in corticospinal excitability after 1800 pulses of subthreshold $5 \mathrm{~Hz}$ repetitive TMS to the primary motor cortex. Clin Neurophysiol 115:1519-1526.

Prut Y, Fetz EE (1999) Primate spinal interneurons show pre-movement instructed delay activity. Nature 401:590-594.

Rajkowska G, Goldman-Rakic PS (1995) Cytoarchitectonic definition of prefrontal areas in the normal human cortex: II. Variability in locations of areas 9 and 46 and relationship to the Talairach coordinate system. Cereb Cortex 5:323-337.

Ridderinkhof KR, van den Wildenberg WP, Segalowitz SJ, Carter CS (2004) Neurocognitive mechanisms of cognitive control: the role of prefrontal cortex in action selection, response inhibition, performance monitoring, and reward-based learning. Brain Cogn 56:129-140.

Riddle CN, Baker SN (2010) Convergence of pyramidal and medial brain stem descending pathways onto macaque cervical spinal interneurons. J Neurophysiol 103:2821-2832.

Riddle CN, Edgley SA, Baker SN (2009) Direct and indirect connections with upper limb motoneurons from the primate reticulospinal tract. J Neurosci 29:4993-4999.

Risterucci C, Terramorsi D, Nieoullon A, Amalric M (2003) Excitotoxic lesions of the prelimbic-infralimbic areas of the rodent prefrontal cortex disrupt motor preparatory processes. Eur J Neurosci 17:1498-1508.

Rowe JB, Toni I, Josephs O, Frackowiak RS, Passingham RE (2000) The prefrontal cortex: response selection or maintenance within working memory? Science 288:1656-1660.

Rushworth MF, Johansen-Berg H, Gobel SM, Devlin JT (2003) The left parietal and premotor cortices: motor attention and selection. Neuroimage 20 [Suppl 1]:S89-S100.

Rushworth MF, Mars RB, Summerfield C (2009) General mechanisms for making decisions? Curr Opin Neurobiol 19:75-83.

Sawaguchi T, Yamane I, Kubota K (1996) Application of the GABA antagonist bicuculline to the premotor cortex reduces the ability to withhold reaching movements by well-trained monkeys in visually guided reaching task. J Neurophysiol 75:2150-2156.

Schluter ND, Rushworth MF, Passingham RE, Mills KR (1998) Temporary interference in human lateral premotor cortex suggests dominance for the selection of movements. A study using transcranial magnetic stimulation. Brain 121:785-799.

Schluter ND, Krams M, Rushworth MF, Passingham RE (2001) Cerebral dominance for action in the human brain: the selection of actions. Neuropsychologia 39:105-113.

Schumacher EH, Cole MW, D’Esposito M (2007) Selection and maintenance of stimulus-response rules during preparation and performance of a spatial choice-reaction task. Brain Res 1136:77-87.

Sheliga BM, Kodaka Y, FitzGibbon EJ, Miles FA (2006) Human ocular following initiated by competing image motions: evidence for a winnertake-all mechanism. Vision Res 46:2041-2060.

Sumner P, Edden RA, Bompas A, Evans CJ, Singh KD (2010) More GABA, less distraction: a neurochemical predictor of motor decision speed. Nat Neurosci 13:825-827.

Tandonnet C, Garry MI, Summers JJ (2011) Selective suppression of the incorrect response implementation in choice behavior assessed by transcranial magnetic stimulation. Psychophysiology 48:462-469.

Taylor PC, Nobre AC, Rushworth MF (2007) Subsecond changes in top down control exerted by human medial frontal cortex during conflict and action selection: a combined transcranial magnetic stimulation electroencephalography study. J Neurosci 27:11343-11353.

Terao Y, Furubayashi T, Okabe S, Mochizuki H, Arai N, Kobayashi S, Ugawa Y (2007) Modifying the cortical processing for motor preparation by repetitive transcranial magnetic stimulation. J Cogn Neurosci 19:15561573.

Touge T, Taylor JL, Rothwell JC (1998) Reduced excitability of the corticospinal system during the warning period of a reaction time task. Electroencephalogr Clin Neurophysiol 109:489-495.

Touge T, Gerschlager W, Brown P, Rothwell JC (2001) Are the after-effects of low-frequency rTMS on motor cortex excitability due to changes in the efficacy of cortical synapses? Clin Neurophysiol 112:2138-2145.

Usher M, McClelland JL (2004) Loss aversion and inhibition in dynamical models of multialternative choice. Psychol Rev 111:757-769.

van den Wildenberg WP, Wylie SA, Forstmann BU, Burle B, Hasbroucq T, Ridderinkhof KR (2010) To head or to heed? Beyond the surface of selective action inhibition: a review. Front Hum Neurosci 4:222. 